\title{
The peculiar binary V Sagittae: Properties of its long-term light changes $^{\star}$
}

\author{
V. Šimon ${ }^{1}$ and J.A. Mattei ${ }^{2}$ \\ 1 Astronomical Institute, Academy of Sciences, 25165 Ondřejov, Czech Republic \\ 2 AAVSO, 25 Birch Street, Cambridge, Massachusetts, 02138-1205, U.S.A.
}

Received September 28, 1998; accepted June 9, 1999

\begin{abstract}
Analysis of the AAVSO and AFOEV visual data (more than 6000 one-day means) revealed that V Sge displayed intervals of the suppressed brightness variations (flat segments), interchanging with intervals of the pronounced changes (active segments). The borderlines of the segments are sharp and are just several days long while the length of the segment is several years. The character of the brightness variations in the active segments evolves and depends on the mean level of brightness in the given segment. The low level gives rise to the separated relatively narrow outbursts while the high state/low state transitions occur in segments with higher mean brightness. We discuss the long-term activity in terms of the divergent models for $\mathrm{V}$ Sge and conclude that the mass accreting white-dwarf primary and variable mass transfer offer a viable explanation for the photometric activity of V Sge. Our analysis therefore brings an additional support to the model by Williams et al. (1986) and Patterson et al. (1998).
\end{abstract}

Key words: stars: activity - binaries: close circumstellar matter - novae, cataclysmic variables — stars: individual: V Sge

\section{Introduction}

V Sge is a close binary $\left(P_{\text {orb }}=0.514 \mathrm{~d}\right)$ with peculiar spectroscopic and photometric characteristics (Herbig et al. 1965, hereafter HPSP). The large amount of circumstellar matter around V Sge manifests itself by very strong complicated emission lines and precludes resolution of any photospheric absorption line (HPSP, Robertson et al.

Send offprint requests to: V. Šimon: simon@asu.cas.cz

* This research has made use of the AAVSO and AFOEV databases.
1997; Patterson et al. 1998; Gies et al. 1998). Also the orbital light curve and its variations are very likely affected by this matter. Due to these circumstances any direct analysis of the components of V Sge (and even a pure determination of the system configuration) is very difficult. There are still two basically different models for V Sge: (a) Algol-type binary, consisting of two non-degenerate stars, the lobe filling primary and detached secondary (HPSP), modified by Wood \& Lockley (1997) who argued that V Sge is a colliding-wind binary; (b) white-dwarf (WD) primary accreting mass through a disk (Williams et al. 1986; Patterson et al. 1998; Steiner \& Diaz 1998); V Sge would be a candidate for the super-soft X-ray source (see van den Heuvel et al. 1992 for definition).

The gradual decrease of the orbital period of V Sge may be due to the mass transfer (Smak 1995; Šimon 1996a; Mader \& Shafter 1997; Patterson et al. 1998). The rate of its change is small, of the order of $\Delta P / P \approx 10^{-10}$ day $^{-1}$. Here it must be admitted that the mass transfer rate quoted by Simon (1996a) is in error. The correct value is near that given by Smak $(1995)\left(\dot{m} \approx 10^{-7} M_{\odot} \mathrm{yr}^{-1}\right)$.

$\mathrm{V}$ Sge has been detected in a broad spectral range, from X-ray (Eracleous et al. 1991; Hoard et al. 1996) and UV (Koch et al. 1986) to radio (Lockley et al. 1997). The latter observations imply a very high mass transfer or outflow rate of the order of $10^{-6} M_{\odot} \mathrm{yr}^{-1}$. Very strong photometric activity has attracted most attention (HPSP, Śimon 1996b - hereafter Paper I, Robertson et al. 1997). In this paper, a preliminary version of which was presented by Šimon \& Mattei (1998), we will further analyse mainly the overall characteristics and long-term evolution of this activity. We will also try to utilize the character of the long-term changes for putting $\mathrm{V}$ Sge into broader context of the interacting binaries. 


\section{The sources of the data}

Securing the data for an analysis of the long-term light changes often represents a big problem because most photoelectric measurements, although of superior accuracy, cover just limited time intervals. The situation is better in the case of the photographic surveys but photographic emulsions are being changed occasionally. On the other hand, monitoring of many variable stars, organized by associations of observers, has made long series of data available. These observations are mostly visual but since they come from a large number of observers, the objectivity of the features of the light curve can be assessed. Visual data, if treated carefully, can be very useful for analysis of long-term activity. Percy et al. (1985) and Richman et al. (1994) discussed the advantages of using visual data and evaluated their accuracy. They found that although the typical error of a single visual observation is about $0.2 \mathrm{mag}_{\text {vis }}$ a much better accuracy of $0.02 \mathrm{mag}_{\text {vis }}$ can be achieved by averaging the data. This accuracy is quite sufficient for analyses of large-amplitude variable stars as V Sge.

Most visual data used in this analysis were obtained from the American Association of Variable Star Observers (AAVSO) International database (Mattei 1996). The original file contained about 25000 measurements, covering the years 1961-1995. The coverage is so dense that strings of multiple observations are sometimes available for a single night. The interval covered by the AAVSO observations was extended by inclusion of a part of the data from the Association Francaise des Observateurs d'Etoiles Variables (AFOEV) extending the coverage back to the years 1934-1944 (see also Paper I). The negative, unreliable and several largely deviating observations were rejected. As was revealed by HPSP, the contribution of the orbital modulation (the reflection effect and mainly the primary eclipse) can be appreciable mainly at the epochs when the system is in its low state. The course of the longterm changes is more clearly defined when this modulation is suppressed by rejecting the data inside the phase interval $0.9-1.1$ (the primary eclipse - see also Paper I). The orbital phase of each observation was calculated using the ephemeris of HPSP since it plausibly satisfies the data in the analysed interval (Smak 1995). We note, however, that the remaining orbital modulation can still contribute to the scatter (see Fig. 7 in HPSP). The data within the phases $0.1-0.9$ were binned into one-day means. The main reason for doing this was that the numbers of observations largely differ for both the respective nights and segments. It would introduce a bias into the statistical analysis. Binning into one-day means is very helpful here because we are interested in the activity on the time scale longer than one day. Moreover, these one-day means were found to display the course of most variations very well.

Table 1 includes the average number of observations $M_{\mathrm{d}}$ in the one-day mean and the coverage of the light

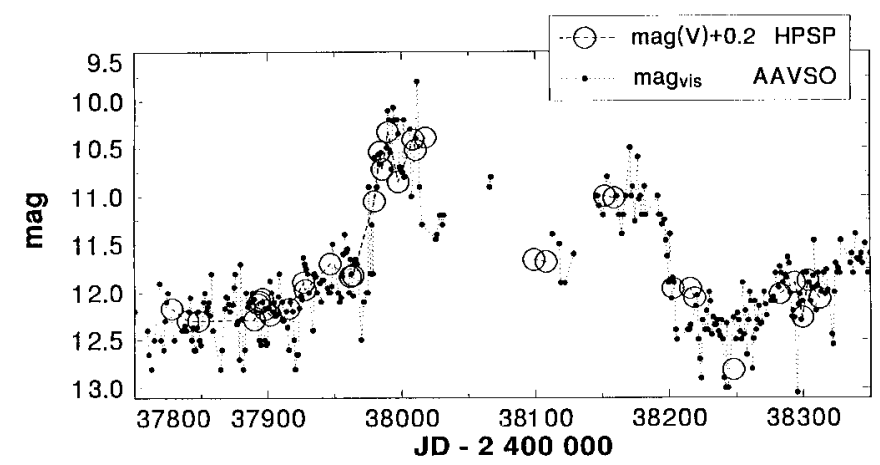

Fig. 1. Comparison of the AAVSO visual data (one-day means) with the out-eclipse photoelectric observations, obtained by HPSP. See Sect. 2 for details

curve (number of one-day means divided by the length of the interval in days) in the respective segments, which are defined below. We can see that most points in the light curve were constructed from multiple observations. We should note that this definition of coverage is of great importance for rapid changes. Inspection of densely populated parts of the light curve shows that the brightness variations, analysed here, occur on the time scale of at least several days, or even weeks, therefore only the seasonal gaps are likely to affect the light curve. If only the seasonal gaps are considered, then the coverage is as high as 40 to 65 percent even in the worst case (segment S0).

The accuracy of the one-day means can be assessed from the scatter of the out-eclipse data. We determined the standard deviations for the means, computed from at least two observations. Typical standard deviation and rms error of a one-day mean during an interval of relatively flat light curve are about $0.15 \mathrm{mag}_{\text {vis }}$ and $0.09 \mathrm{mag}_{\mathrm{vis}}$, respectively, but one should bear in mind that the error includes both observational inaccuracies and intrinsic variations (mainly the orbital modulation). Another way to assess the reliability of the visual light curve is a comparison with photoelectric observations. We show the oneday visual means with superposed photoelectric observations, obtained by HPSP, in Fig. 1. It can be seen that the observations obtained out of eclipse by both methods are in good agreement and that the visual data give a good description of the long-term variations. We found that a slight systematic shift of the photoelectric data by $0.2 \operatorname{mag}(V)$, applied also in Fig. 1, improves the match. This effect may be explained by slightly different passbands of the $V$ filter and the eye. We will therefore abbreviate the brightness determined from the visual data as mag vis. $_{\text {. }}$.

The CCD observations in the high state by Robertson et al. (1997) show occasional rapid dips with an amplitude about $1 \operatorname{mag}(V)$ and duration at most 10 days. However, we will concentrate just on the long-term changes which 


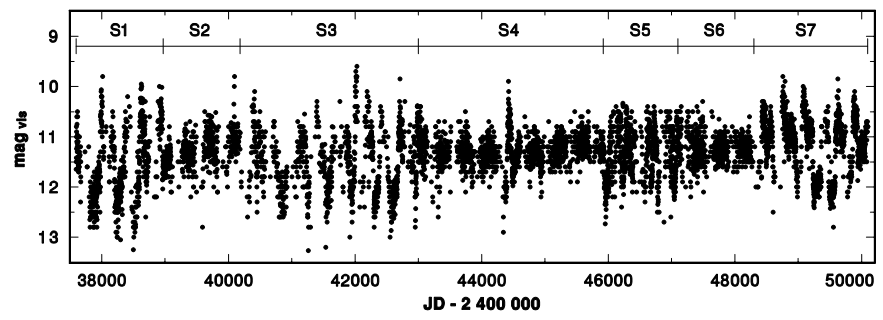

Fig. 2. The light curve of V Sge, constructed from the AAVSO one-day means of observations, displayed on the condensed time scale and covering the years 1961-1995. The respective segments, discussed in the text, are marked by S1-S7

of course are clearly visible in a series of visual data, not to overinterpret the visual data and work with the noise.

\section{Analysis of the data}

\subsection{General description}

The whole light curve, constructed from the AAVSO oneday means (about 6070) of observations, is displayed on a condensed time scale in Fig. 2. The respective parts, plotted on an expanded time scale, can be seen in Fig. 3 and Fig. 4. The seasonal gaps are usually short and the dense coverage allows to follow the course of the brightness variations almost day by day. One can immediately resolve that the amplitude significantly varies on the time scale of years. There are definitely groups of large variations with an amplitude about 2 mag $_{\text {vis }}$. These changes have a repeating character and occur on a typical time scale of hundreds of days. On the other hand, extended intervals (years) of a flat light curve with just minor fluctuations can be seen in other years.

The AAVSO data set begins with an epoch of largeamplitude variations (from $10 \mathrm{mag}_{\text {vis }}$ to $13 \mathrm{mag}_{\mathrm{vis}}$ ). The extrema of brightness are sharp, especially the maxima have a character of distinct peaks ("outbursts"). Both gradual and abrupt transitions between extrema can be resolved. Notice that after 3.8 years this season abruptly ends around JD $=2438970$ with a transition into an intermediate level of brightness (about $11 \mathrm{mag}_{\mathrm{vis}}$ ); a brief dip may have occurred here. This transition can be established with an accuracy of several days.

$\mathrm{V}$ Sge spends the next 3.3 years in this intermediate level. Just minor variations can be seen till JD $=2440190$ when a rapid decline of brightness introduced another series of large-scale variations. In this season which lasted for 8 years (till JD $=2443000$ ) the brightness varied from about $10.5-11 \mathrm{mag}_{\text {vis }}$ to $12.5 \mathrm{mag}_{\text {vis }}$ (several brief excursions up to about $10 \mathrm{mag}_{\text {vis }}$ ). Most extrema of brightness are flatter and more rounded now. The light curve is gaining a character of alternating high and low states in this season (hereafter HS and LS). Part of this segment was already plotted with smaller time resolution in Fig. 5 of Paper I, but owing to weaker coverage the AFOEV data were averaged over several days. Despite the heavier smoothing, which left only the long-term changes in the AFOEV data, the mean course of the HS/LS variations is in agreement with the AAVSO observations.

Another extended interval of an almost flat light curve, lasting for about 8 years, spans JD $=2443000-2445900$ The interval is interrupted near JD $=2444400$ by one event, having the character of an isolated outburst. Its analysis was given in Paper I. The duration of this outburst is much shorter than the surrounding interval of the flat light curve.

The next season of large-scale variations, which was introduced by a well defined rapid decline into a low state, set in near JD $=2445900$. Most variations with an amplitude larger than about $1 \mathrm{mag}_{\text {vis }}$ can be described as alternating HSs and LSs again. However, they occur on a shorter time scale (up to 100 days) than in the previous season of the HS/LS transitions. Besides the episodes of well defined HSs and LSs one can resolve short-term variations in the high state; they take place on the time scale of days and have an amplitude of several tenths of $\operatorname{mag}_{\text {vis }}$. The alternating HSs and LSs vanished around $\mathrm{JD}=2447300$ and the brightness remained near the level of HS. Although some fluctuations of brightness are present after this date (till about 2447500 ) they do not resemble the episodes of LSs anymore. Brightness of V Sge showed no episodes of low state till JD $=2448300$ (for about 3 years).

Although some data are missing near JD $=2448300$ it is evident that an episode of LS occurred here and introduced a new extended series of alternating HSs and LSs. These states are very pronounced now and repeat on the time scale of 200-300 days. Most transitions between the states are relatively abrupt (i.e. much shorter than the duration of the state) and can be easily resolved. The high states usually display some structure. Brightness of a HS immediately after recovery from a LS is often higher than that before the decline into LS. Also some shallow minimum near the middle of a HS can be resolved. Detail of the brightness fluctuations in HS is shown in Fig. 8 in Paper I, again averaged over several nights.

In summary, this examination of the AAVSO light curve revealed that the seasons of the suppressed brightness variations (hereafter called flat segments) interchange with intervals of pronounced changes (hereafter active segments). The borderlines of the segments are usually well defined and the light curve can be divided into seven seasons. They are marked in Fig. 2 and abbreviated as S1-S7. Changes of brightness in the active segments often have a character of the HS/LS transitions. The flat segments display just minor fluctuations of brightness and although some rare larger excursions (a few days) from the main level may be present, they can better be compared to the brief dips, documented by Robertson et al. (1997). 

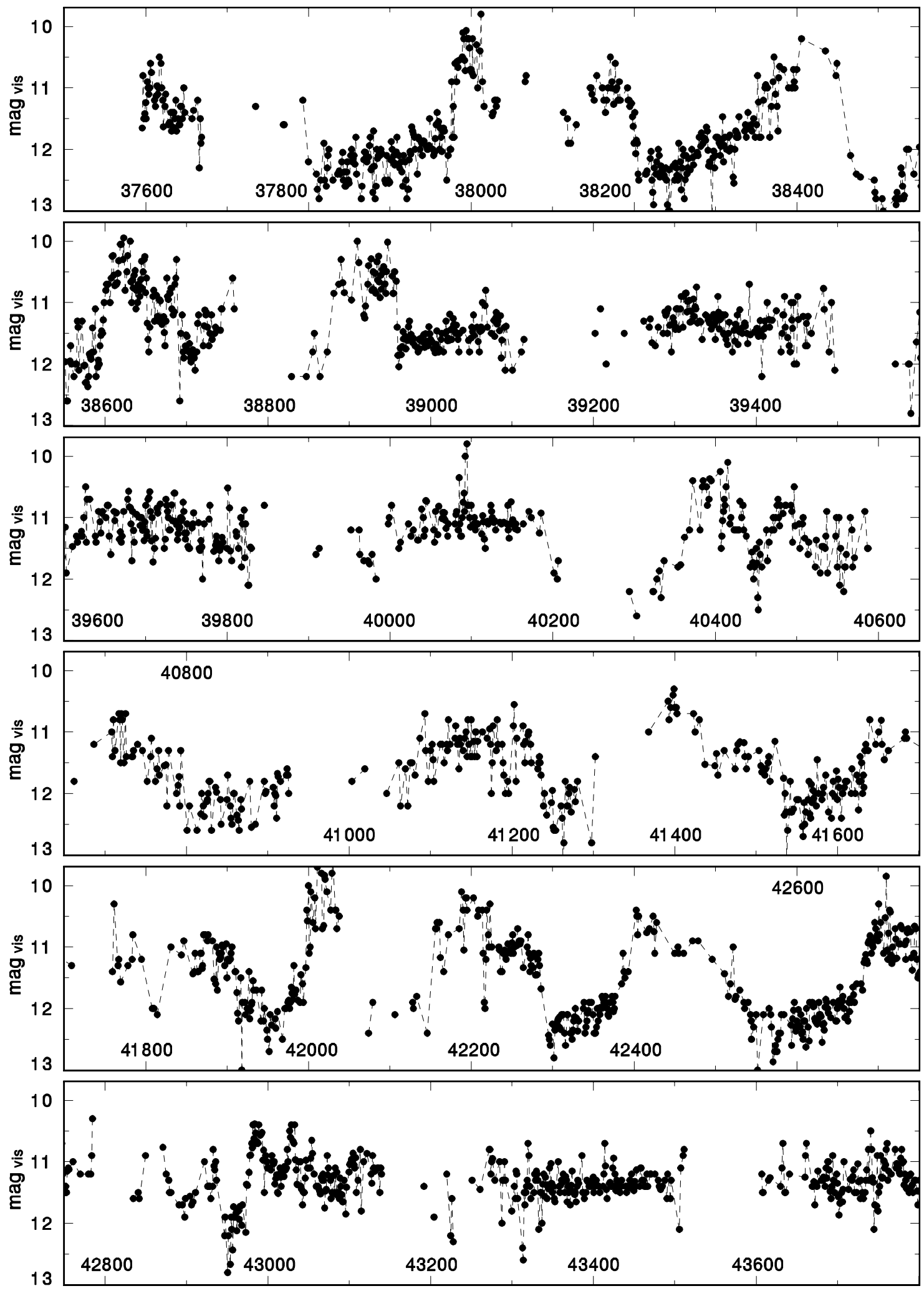

Fig. 3. The same AAVSO light curve as in Fig. 2 but plotted with high temporal resolution (the first half) 

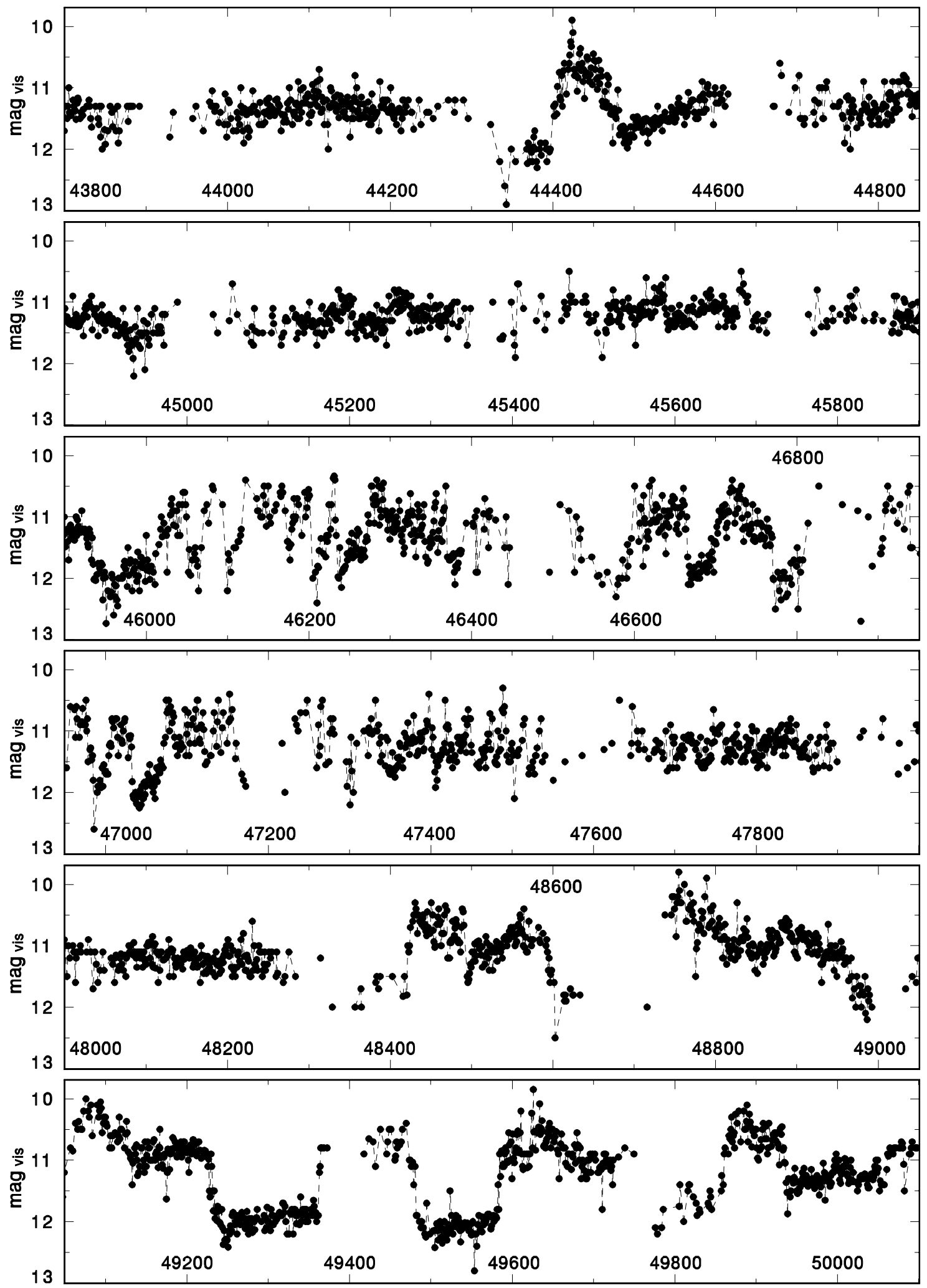

Fig. 4. The same AAVSO light curve as in Fig. 2 but plotted with high temporal resolution (the second half) 

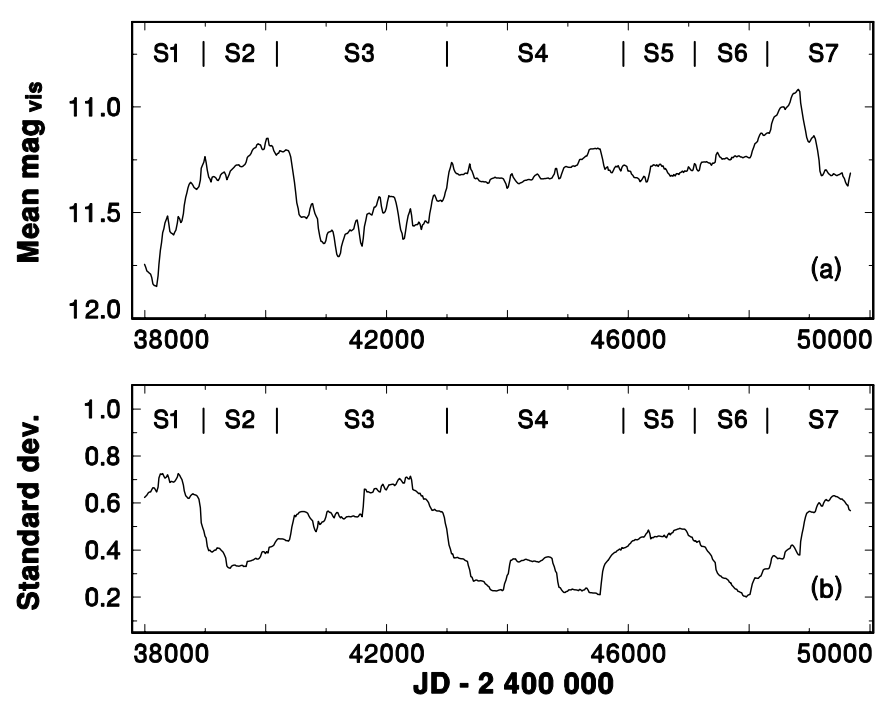

Fig. 5. a) Two-sided moving averages of the brightness for the AAVSO data. b) Standard deviations $\sigma_{\text {mag }}$ of the moving average of brightness. Result for the parameter $Q=400$ days is shown. See Sect. 3.2 for details

Our data do not allow to carry out a reasonable assessment of such short rare events.

One more segment, formed from the AFOEV data and representing the light curve in the years 1934-1944, is included. Its large part is displayed in Fig. 2 in Paper I and can be characterized as a long-lasting epoch of low brightness (near $12 \mathrm{mag}_{\mathrm{vis}}$ ) with occasional relatively narrow outbursts (see Paper I for details). In the following analysis we will treat it as segment S0. We note that there are just very few scattered data within the years 1944-1961 and do not enable any reasonable analysis in this time period.

\subsection{Moving averages}

Moving averages is one method which enables us to suppress the high frequency variations and obtain thus a better insight into the long-term trends. We will present the results of this method first because it allows to analyse variations through the whole data set without further presumptions. Two-sided moving averages of the AAVSO data were calculated for several values of $Q$. The parameter $Q$ refers to the interval of days, within which the data were averaged. We also introduce the standard deviation $\sigma_{\text {mag }}$ of every calculated mean point. This quantity gives information about the current amplitude of the variations.

We calculated the moving averages for several values of $Q$. Figure 5 displays the results for $Q=400$ days. This filter half-width washed out the variations inside the active segments and emphasized the general levels of brightness. 200 to 570 observations are inherent in each mean. It can be resolved in Fig. 5a that the lowest mean brightness occurs in S1 but increases towards the transition from S1 to $\mathrm{S} 2$ (from active to flat segment) near JD $=2439000$. Also initially large $\sigma_{\text {mag }}$ rapidly decreases towards $\mathrm{S} 2$. The average level of brightness in S3 (active segment with HSs and LSs) is clearly lower than in the neighbouring flat segments $\mathrm{S} 2$ and $\mathrm{S} 4 . \sigma_{\mathrm{mag}}$ slightly grows through segment S3 but then rapidly falls as the border with $\mathrm{S} 4$ approaches.

The active segment S5 is clearly defined by a large bump in $\sigma_{\text {mag }}$ (Fig. 5b). However, the light curve in Fig. 5a shows that the mean brightness level through this segment stayed almost unchanged with respect to adjoining S4 and S6.

HSs and LSs in S7 are very pronounced and have a long cycle-length. They therefore tend to be more sensitive to the seasonal gaps. The course of the moving averages for this segment may be then distorted to some extent. Nevertheless, the mean light curve shows that the highest brightness did occur in this segment.

In summary, the moving averages reveal the long-term changes of the mean level of brightness of V Sge. Mainly the respective seasons of the large-amplitude variations (manifested by enhanced $\sigma_{\text {mag }}$ and corresponding to the active segments) tend to brighten with time; this is clear especially for the sequence S1-S3-S5. The course of $\sigma_{\text {mag }}$ confirms the obvious fact that amplitude of the brightness changes varies in agreement with the division into segments. We note that the transitions between the neighbouring segments are inevitably rounded in Fig. 5a,b due to the large value of $Q$ used.

\subsection{Statistical distributions of brightness in the segments}

We have argued above that the light curve of V Sge can be divided into segments with the well defined limits. The dense coverage ensures that this division is not oversimplified. When the data set displays seasons of such clearly divergent behaviour then it is a reasonable approach to analyse each segment separately. It also gives an opportunity to search for the overall characteristics of the activity in a given interval. The segment can be characterized by its net parameters (mean brightness, median, skewness, excess). These net parameters can be used for an assessment of the evolution of the long-term activity.

The histograms of the one-day means, constructed for the brightness in segments S0-S7, are displayed in Fig. 6. The width of each bin is 0.25 mag $_{\text {vis }}$ in all cases. Parameters of the distributions can be found in Table 1. The distribution of the whole AAVSO data set (S1-S7) is clearly unimodal without any other features and is roughly symmetrical. We preferred not to include segment S0 in this histogram since it represents a season separated from the rest of the data by a gap of about 17 years.

Large changes of the distribution from segment to segment can be seen when the respective histograms are 

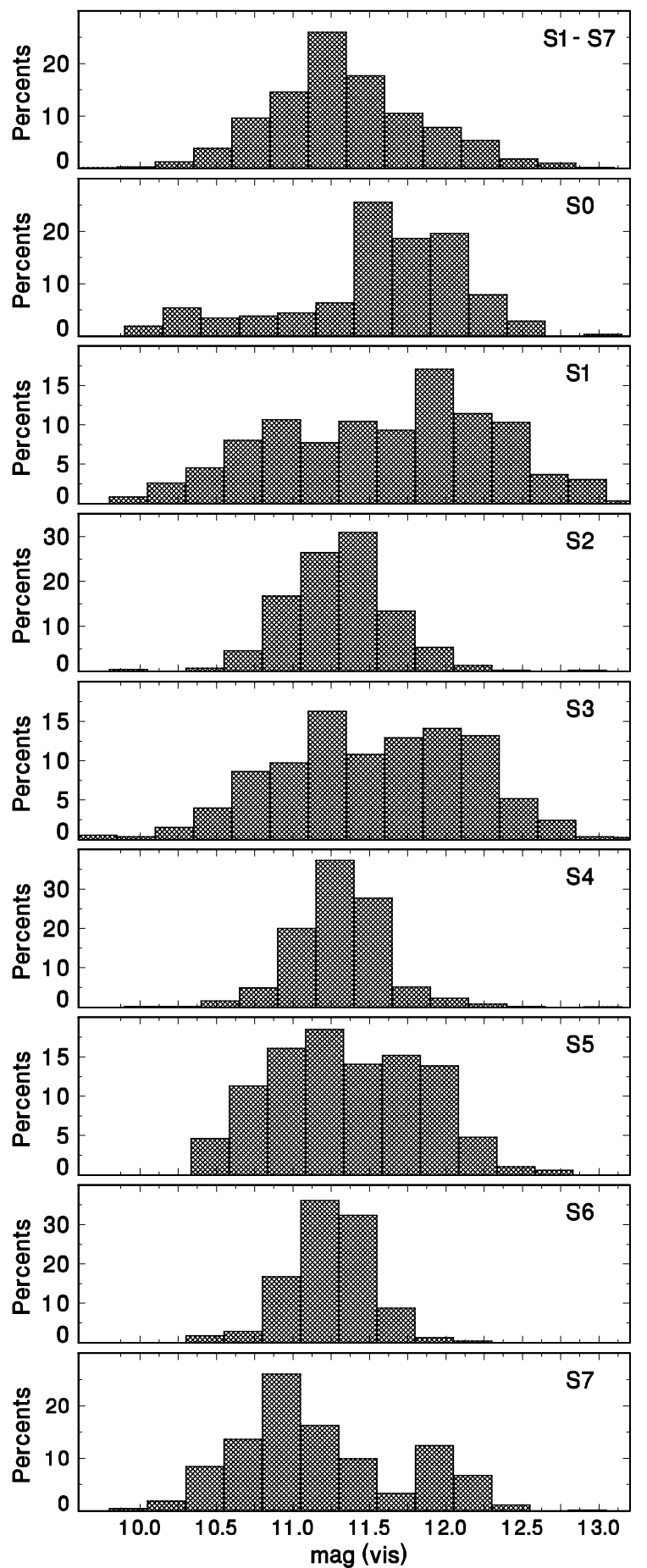

Fig. 6. The statistical distributions of brightness constructed from the one-day means of observations in the respective segments. The intervals in JD and parameters of the segments are given in Table 1

constructed for S1 to S7. They confirm the division presented above. Notice three similar segments S2, S4, S6 which are clearly unimodal, relatively narrow, roughly symmetrical and all have almost identical mean levels (near $\left.11.3 \mathrm{mag}_{\text {vis }}\right)$. On the other hand, very broad distributions are observed in active S1, S3, S5, S7. The histogram for $\mathrm{S} 1$ is flat and has an asymmetrically placed peak near $11.9 \mathrm{mag}_{\mathrm{vis}}$. The distributions of S3 and S7 are bimodal. The histogram for S5 is slightly more narrow than S3 and S7 and its bimodality is at most marginal.

\subsubsection{Evolution of the brightness changes}

The divergent distributions, apparent in the histograms for the individual segments, suggest changes on a very long time scale (years to decades) in the activity of V Sge. The net parameters of each segment (mean brightness, skewness, excess), listed in Table 1 and plotted versus JD in Fig. 7, allow for a better insight into these trends.

The evolution of the net parameters of the active segments S0, S1, S3, S5, S7 deserves special attention. The most striking variation is a gradual change of asymmetry of the histograms (skewness) in the sequence S0-S1S3-S5-S7 (Fig. 7a). This change through the four latter segments can be considered linear; the fit is denoted by dashed line in Fig. 7a. We admit that the limits of segment S0 are not so clearly defined as the rest of the segments. Nevertheless, skewness of S0 follows the trend of the later active segments.

A gradual increase of the mean brightness in the sequence S1-S3-S5-S7, apparent in Fig. 7c, amounts about $0.4 \mathrm{mag}_{\mathrm{vis}}$. The points are connected by a line for clarity. Mean levels of HSs and LSs are included; they allow to resolve their contribution to the resulting trend. It turns out that the mean levels of HSs in active segments are always brighter than the levels of the adjacent flat segments S2, S4, S6. Brightening of the active segments is in accordance with the moving averages (compare Fig. 5a and Fig. 7c). Two levels of LS in S7 are displayed, with the last episode and without it (see below).

Comparison of Fig. 7 with Figs. 2, 3 and 4 allows to relate evolution of the net parameters with the changes of the character of the light curve in the respective active segments. The large negative skewness and low mean brightness of S0 are caused by the relatively narrow outbursts from the quiescence level (Fig. 2 in Paper I). The type of variations in segment $\mathrm{S} 1$ is intermediate between the separated outbursts in S0 and HS/LS transitions in S3, S5, S7. The well defined transitions between HS and LS, analysed in Sect. 3.4, began in S3. With increasing mean brightness in the next active segments V Sge spends more time in HS than in LS, causing thus an increase of skewness.

\subsection{Characteristics of the HS/LS transitions}

The alternating high and low states are a characteristic photometric activity of several kinds of interacting binaries: cataclysmic variables $(\mathrm{CVs})$ of the VY Scl type (e.g. Shafter et al. 1985), polars (e.g. Hudec \& Meinunger 1977), X-ray binaries (e.g. Hudec \& Wenzel 
Table 1. Parameters of the respective segments of the light curve of V Sge. Intervals of JD-2 400 000, duration (in days) and coverage by the one-day means (in \%) of the respective segments (S) are given along with the mean visual magnitude, its median, skewness and excess. S1-S7 are identified in Fig. 2 (AAVSO data) while S0 is formed from the AFOEV data (see the text). Standard deviation $\sigma_{\text {mag }}$ of the brightness in the whole segment is included. Number of the night means in the segment is given in the column $N$ (obs). $M_{\mathrm{d}}$ refers to the average number of observations in the one-day mean

\begin{tabular}{rrrrrrrrrrr}
\hline $\mathrm{S}$ & Interval of JD & Duration & $N$ (obs) & $M_{\mathrm{d}}$ & Cover. & Mean mag & Median & $\sigma_{\text {mag }}$ & Skewness & Excess \\
\hline S0 & $27678-31438$ & 3760 & 317 & 1.2 & $8 \%$ & 11.54 & 11.60 & 0.58 & -0.88 & 0.40 \\
S1 & $37595-38969$ & 1374 & 621 & 3.2 & $45 \%$ & 11.58 & 11.70 & 0.70 & -0.21 & -0.78 \\
S2 & $38970-40173$ & 1203 & 465 & 7.6 & $39 \%$ & 11.28 & 11.30 & 0.34 & 0.02 & 1.38 \\
S3 & $40183-42999$ & 2816 & 927 & 2.2 & $33 \%$ & 11.52 & 11.50 & 0.62 & -0.18 & -0.46 \\
S4 & $43001-45919$ & 2918 & 1590 & 3.0 & $54 \%$ & 11.29 & 11.30 & 0.29 & 0.17 & 2.32 \\
S5 & $45920-47099$ & 1179 & 691 & 2.6 & $59 \%$ & 11.35 & 11.32 & 0.49 & 0.14 & -0.78 \\
S6 & $47100-48283$ & 1183 & 640 & 2.2 & $54 \%$ & 11.22 & 11.20 & 0.26 & -0.20 & 0.81 \\
S7 & $48314-50097$ & 1783 & 1133 & 2.8 & $64 \%$ & 11.15 & 11.03 & 0.54 & 0.45 & -0.59 \\
All(S1-S7) & $37595-50097$ & 12502 & 6067 & 3.1 & $49 \%$ & 11.33 & 11.30 & 0.50 & 0.34 & 0.29 \\
\hline
\end{tabular}

1986). However, due to its short duration, only rarely is the course of the transition between the states covered by the observations. V Sge displayed the typical HS/LS transitions in active segments S3, S5, S7 (see Sect. 3.1). Dense coverage of the AAVSO data allowed to resolve the course of these transitions in 36 cases. The typical appearance of the HS/LS variations in V Sge can be described as follows. The onset of the episode of LS begins with a rapid fall of brightness from HS by about $1 \mathrm{mag}_{\text {vis }}$. The phase of LS is not quite flat in some cases; it displays a very slow brightening (e.g. LSs centered on JD = 2449300 and 2449 520). The final return to HS is approximately as rapid as the decline. Brightness after return to HS is sometimes (mainly in S7) slightly higher than immediately before onset of LS. Interchanging HSs and LSs in segment S5 occurred on a short time scale (Sect. 3.5) and the stable level of LSs was not always fully developed.

The declining and rising parts of the light curve of the well covered transitions were approximated by straight lines. Linear least squares fits were used inside the intervals, adjusted interactively, for determination of the parameters of the transitions. The results for S3, S5 and S7 are summarized in Table 2 .

Not all transitions have equal amplitude; many states, especially HSs, display their own structure (fluctuations on the time scale of days or trends-often brightening through LS and decline through HS). The transition therefore does not always begin and finish in the mean level of the state. In order to describe the transitions in S3, S5, S7 fully, both duration and the rate of change $\Delta \mathrm{mag} / \Delta t$ are needed (Fig. 8ab). The lower limit of the duration is comparable for all three segments (typically several days, the shortest transition only 4 days). The respective segments largely differ mainly in the range over which the durations are scattered. Transitions in S3 are generally slow with a large scatter ( 7 to 80 days), which also is reflected in their low rate of change. On the contrary, S5 represents a relatively tight group of very rapid transitions (4 to 11 days). The rate of change for the respective transi-
Table 2. Parameters of the well covered HS/LS transitions in segments S3, S5, S7. The upper two rows give the numbers of the respective measured H-L (from the high to the low state) and L-H (from the low to the high state) transitions analysed. Durations and amplitudes (in $\operatorname{mag}_{\text {vis }}$ ) of the transitions are listed along with their standard deviations

\begin{tabular}{lrrrr}
\hline & S3 & S5 & S7 \\
\hline No. of H-L & 6 & 8 & 3 \\
No. of L-H & 7 & 7 & 5 \\
Dur. H-L (days) & $33.5 \pm 25.4$ & $6.7 \pm 2.3$ & $21.3 \pm$ & 7.4 \\
Dur. L-H (days) & $25.1 \pm 16.7$ & $8.3 \pm 1.7$ & $13.2 \pm 8.2$ \\
Ampl. H-L (mag) & $1.12 \pm 0.19$ & $1.25 \pm 0.26$ & $1.17 \pm 0.35$ \\
Ampl. L-H (mag) & $1.30 \pm 0.16$ & $1.04 \pm 0.15$ & $1.18 \pm 0.08$ \\
\hline
\end{tabular}

tions in S5 varies by one to four, which is larger than the ratio of the durations and confirms scatter of the amplitudes. Parameters of the transitions in $\mathrm{S} 7$ are intermediate to S3 and S5.

Statistical distribution of durations of all measured transitions (Fig. 9) shows a clustering between 4 and 20 days and a weak tail towards prolonged transitions (up to 80 days).

We further analysed a possibility of gradual variations of the brightness levels of the respective HSs and LSs through the whole active segment. We mainly checked the working hypothesis that the brightness difference between HS and LS is largest near the middle of the segment and decreases towards its borders (possible gradual development and vanishing of the instability). The mean brightness of each state with the removed transitions was calculated, received a unit weight and was centered on the middle of the appropriate state. Inspection of the data confirmed that the level of the given state was achieved also in several cases of weaker coverage. The means were fitted by linear and quadratic polynomials. Upward curvature of the quadratic fit of HSs and downward curvature for LSs would be expected if our working hypothesis were true. 


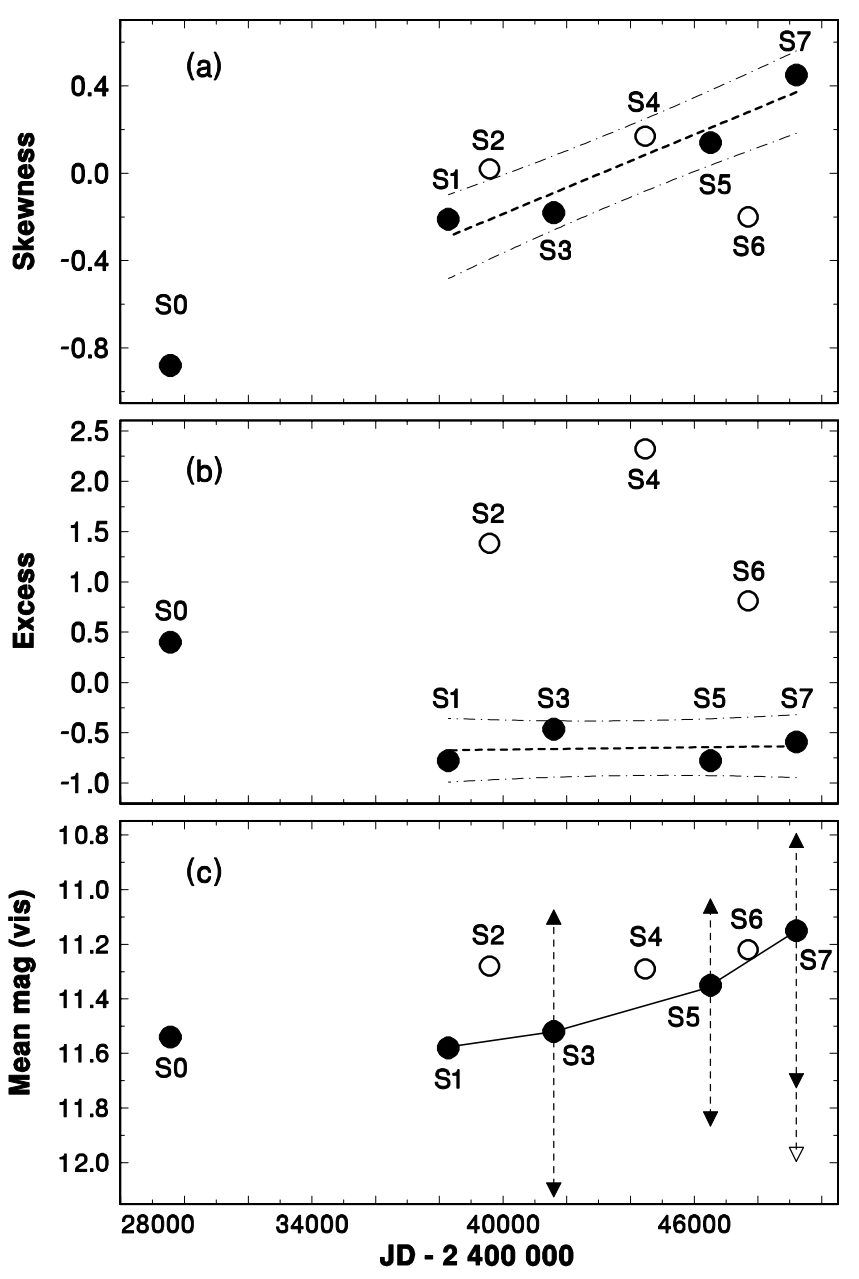

Fig. 7. Net parameters of the respective segments from Table 1 plotted versus Julian Date. Each point is centered on the middle of the appropriate segment. See Sect. 3.3.1 for details

The linear fits both to HSs and LSs satisfy the course well in segment S3; the quadratic terms are very small. However, the brightness difference between HS and LS linearly increases through S3. Notice that this behaviour is also apparent in the evolution of $\sigma_{\text {mag }}$ in Fig. 5 b. Linear fits are justified also in S5. They reveal small decrease of brightness of both HSs and LSs through this segment while the brightness difference between HS and LS stays almost constant. Brightness of HSs increases linearly through S7. At the same time, levels of the respective LSs in S7 display more complicated changes with a prominent increase of brightness during the last two episodes. It supports the interpretation of the medium state after JD $=2449940$ as an episode of an exceptionally shallow LS.

We can conclude that with the exception of LSs in S7 the changes of the levels of the respective states are small and their courses can be considered linear. It strengthens the above given arguments that the transitions between the adjoining segments are rapid and that the characteristics of HSs and LSs are usually kept for the whole duration of the given segment.
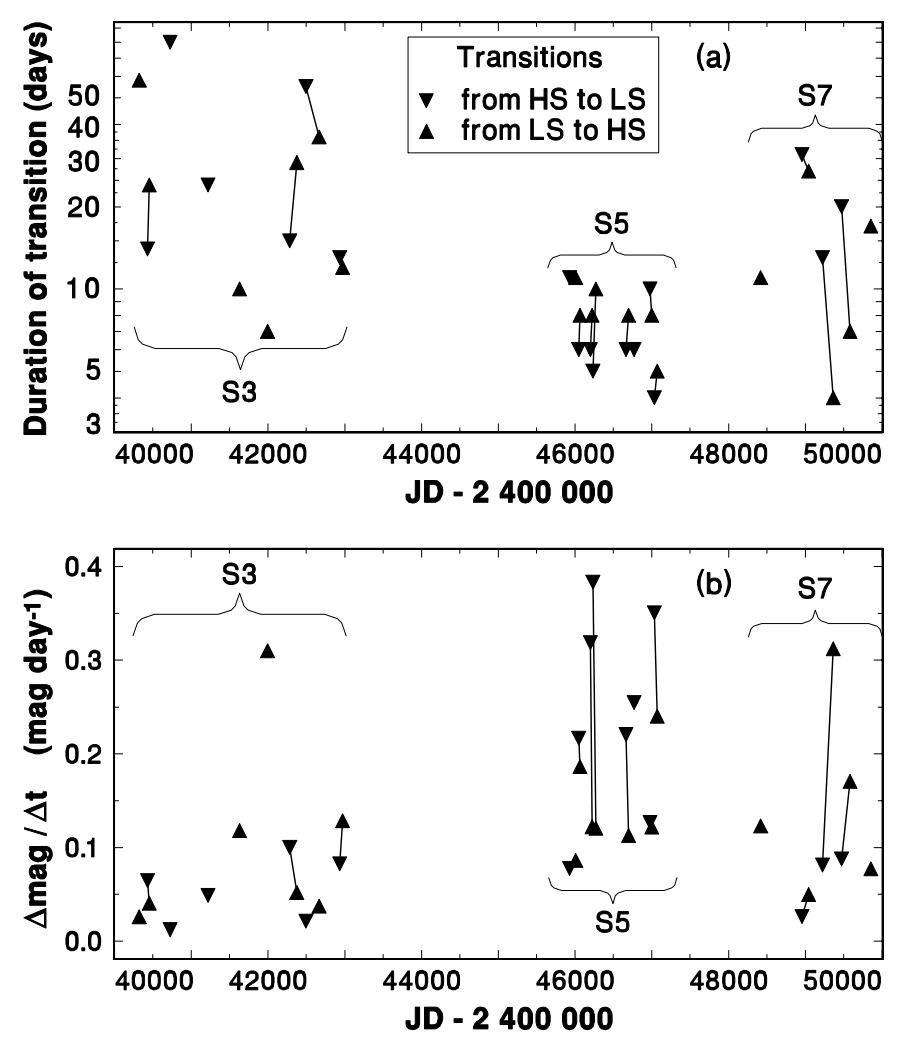

Fig. 8. a) Duration of the respective HS/LS transitions in three active segments (S3, S5, S7) of typical HS/LS behaviour of V Sge, plotted versus Julian Date. Logarithmic scale is used for the $y$-axis. b) Rate of the brightness change during the transitions. Those transitions which belong to the same episode of the low state (fall and rise of brightness) are connected by lines. See Sect. 3.4 for details

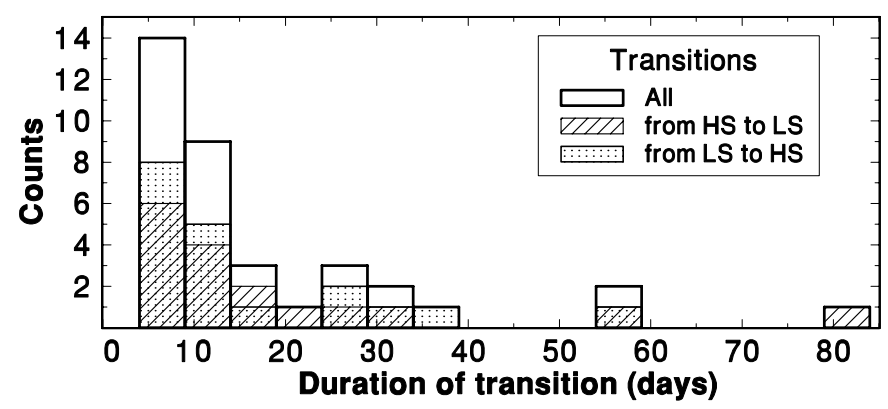

Fig. 9. Histogram of durations of HS/LS transitions in S3, S5, S7

\subsection{Periodicities in the $H S / L S$ transitions}

Already visual inspection revealed that most HS/LS transitions occur in at least a semi-regular manner. A search for periodicities was therefore undertaken using the PDM program and the autocorrelation method. The PDM program ("phase dispersion minimization"), based on the method of Stellingwerf (1978) and written by Dr. J. Horn at the Ondřejov Observatory, is suitable for nonsinusoidal 


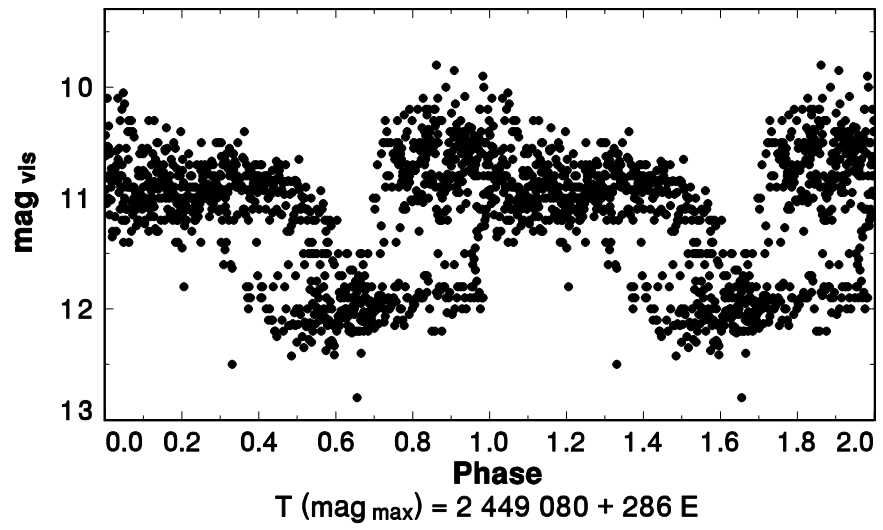

Fig. 10. The data inside the epoch of the HS/LS transitions (segment S7; JD = 2448314-2 449 930) folded with the period of 286 days. The peak at JD $=2449080$, which occurred after recovery from LS, was taken as the initial moment of the folding. See Sect. 3.5 for details

time variations covered by irregularly spaced observations. Significance of a given period is evaluated by the parameter $\Theta$, lying in the range $0-1$. Insignificant periods have $\Theta \approx 1$ while highly significant periods are expected to have $\Theta \approx 0.4$ or lower. Horn's program enables not only an automatic searching for the best period inside a given interval but also an interactive examination of the resulting data foldings. It allows to assess whether the course of the particular folded light curve is reasonable.

The autocorrelation method, described by Percy et al. (1981), allows to search for characteristic time scales or quasiperiods which extend just for several cycles. This method makes use of the brightness difference $\Delta$ mag versus the time difference $\Delta t$ of each observation, divided into equal bins. (Quasi)periodic behaviour then gives rise to the minima in the resulting $\Delta$ mag versus $\Delta t$ curve.

Segment S7 was analysed first because it contains the most pronounced HS/LS transitions. We restricted ourselves to JD $=2448314-2449930$ and excluded the last LS which brightness is roughly intermediate to HS and LS. The period search revealed two possible periods: 286 days $(\Theta=0.684)$ and 238 days $(\Theta=0.807)$. Notice that the latter value is very close to the period of 240 days, found by Robertson et al. (1997) in their CCD data. However, it gives too many points in antiphase. Our data folded with $P=286$ days can be seen in Fig. $10 . \Theta=0.684$ implies that the HS/LS transitions cannot be considered strictly periodic; 286 days is just a typical time scale. The autocorrelation diagram displays a prominent deep minimum at 270 days (Fig. 11) which is in good agreement with the result of the PDM method. We therefore can conclude that the quasiperiod near 280 days has its meaning and is not just accidental because the folded light curve bears much of the course of the original data.

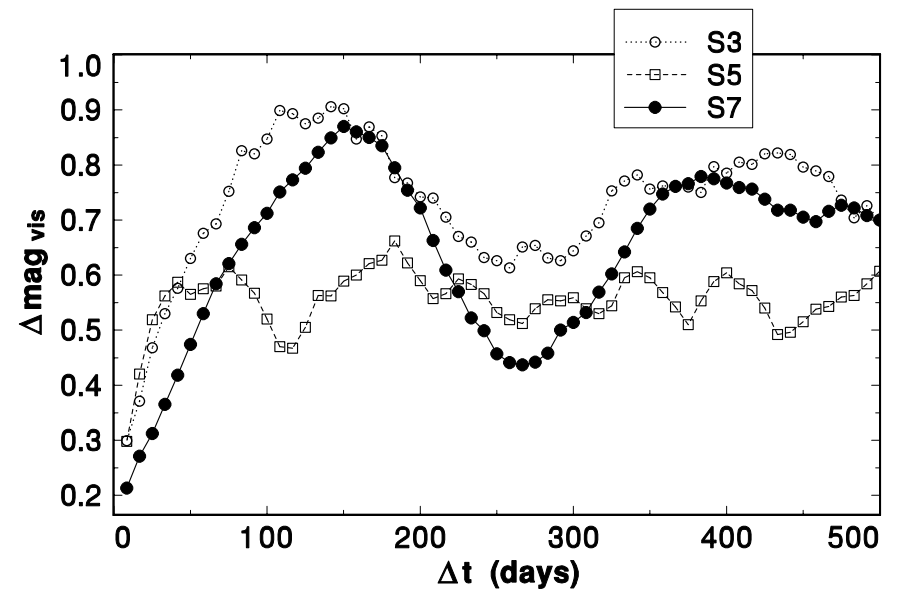

Fig. 11. Autocorrelation diagram for the segments S3, S5, S7. Notice the deep minimum at 270 days for the segment $\mathrm{S} 7$ which is in good agreement with the period of 286 days, found by the PDM program (Fig. 10). See Sect. 3.5 for details

The PDM program did not reveal any period with $\Theta<0.75$ in S3. The autocorrelation diagram displays a minimum at 260 days but it is not as deep as in S7 (Fig. 11).

Inspection of segment S5 revealed that the cycle-length is shorter than in S3 and S7. However, no periods with $\Theta<0.85$ were found. Even the autocorrelation diagram does not display any prominent feature. Part of this segment was also covered by the photographic data, analysed by Marsakova (1998). The dominant period of 61 days, found by Marsakova, may represent a characteristic time scale of the HS/LS transitions, persisting just for several consecutive transitions; it only confirms a shorter cycle of the transitions in this segment.

In summary, only typical cycle-lengths, not strict periods, can be traced in the active segments. We can state that while the HSs and LSs in segments S3 and S7 tend to occur on the time scale of about 270 days, a significantly shorter time scale $(<100$ days) prevails in S5.

\section{Discussion}

We have shown that V Sge displayed interchanging seasons of largely different activity during the last decades. We demonstrated that regularities and trends can be found both for the general features of the light curve and for the main events. The main results can be summarized in the following way:

1. Intervals of the suppressed brightness variations (flat segments) interchange with intervals of the pronounced changes (active segments). The borderlines of the segments are sharp. The lengths of the segments (active versus flat) are comparable and are within about one to three, the length of a segment being several years. 
2. The character of the brightness variations in the active segments evolves and depends on the mean level of brightness in the given segment. The low level gives rise to the separated relatively narrow outbursts while HS/LS transitions occur in segments with higher mean brightness. The system spends more time in HS than in LS and these states are more clearly resolved in the histograms for segments with high mean brightness level. This evolution is quantified by a gradual monotonic variation of skewness of the active segments. HSs are brighter than the levels of the adjacent flat segments.

3. The typical HS/LS variations in segments S3, S5, S7 consist of the well defined levels, separated by relatively rapid transitions with amplitude about 1 mag $_{\text {vis }}$. HSs can show "fine" structure, the resulting course of HS/LS is a superposition of the well defined transitions and variations of brightness through the given state. There are no significant differences between the durations of the rise from LS and the decline from HS. With the exception of LSs in S7 the changes of the levels of the respective states are small and their courses can be considered linear. It suggests that the instability develops rapidly at the onset of the active segment (within a single episode of LS) and that the characteristics of HSs and LSs are usually kept for the whole duration of the given segment.

4. The HSs and LSs occur on a timescale typical for a given segment, though suggested just barely. In any case, the cycle-length near 270 days, found for the HS/LS transitions in S3 and S7, was significantly longer than in S6 ( $<100$ days).

In the following we will confront the observed activity with the mechanisms which are physically acceptable in the respective models for $\mathrm{V}$ Sge. We will also make use of a comparison of the characteristics of V Sge with examples of the relevant classes of objects.

\subsection{Colliding wind Algol-type binary}

Comparison with the activity of the colliding wind binaries (mainly the Wolf-Rayet, WR) reveals that the amplitudes of the brightness variations in the optical are seriously discordant: changes in most WR stars are less than $0.1 \mathrm{mag}(V)$ even on the time scales of decades (Schmutz 1991). There are a few exceptions-changes of the depth of the eclipse in the WR binary CV Ser by up to $0.5 \mathrm{mag}_{\mathrm{y}}$ (Hjellming \& Hiltner 1963) and two dips 1.2 mag deep, separated by 71 years, in HD 164270 (Massey et al. 1984). However, even in these two cases the kind of activity is hardly comparable to V Sge. The character of activity in V Sge also largely differs from the Be stars, in which the circumstellar envelopes and stellar winds play a large role in the brightness variations. Their long-term variations are rather smooth (waves or broad shallow states of lower brightness), have a typical amplitude just of the order of 0.2 to $0.4 \operatorname{mag}(V)$ and occur on the time scale of months and years (e.g. Mennickent et al. 1994; Harmanec 1994).

The characteristics of HSs and LSs in V Sge (distinct levels of brightness, repetition (pronounced in S7) and often rapid transitions between the states-sometimes just 4 days (Figs. 8, 9) put constraints on the underlying mechanism. The circumbinary envelope (uneclipsed light), supposed by HPSP and Mader \& Shafter (1997), can only partly account for the brightness variations in HS/LS transitions. Changes of primary's radius $R_{1}$ (sometimes $R_{1}$ even larger than its lobe), detected by HPSP, must occur near LS, too, in order to ensure the observed amplitude. It suggests that the process in transitions between the levels of HS and LS is complex and would imply a tight interplay between the stellar components (especially the primary) and the envelope (changes of $R_{1}$ near LS leading to the envelope formation). Since there is not significant difference between duration of the transitions from LS to HS and from HS to LS (Table 2), the interplay occurs on the same short time scale for both transitions and suggests a large dependence of the envelope on the current rate of the mass supply. Large abrupt changes of $R_{1}$ during transitions appear unrealistic if we suppose that we see directly the photosphere of the primary. It is difficult to imagine a mechanism which would rapidly alternate radius of this star between two states to produce the brightness variations in HS/LS transitions. Moreover, radius of the lobe is a quite strict limit for the dimension of the star. Instead, we can get a plausible solution if we interpret the visible dimension of the primary not as a photosphere, but as a current radius within which its wind is optically thick. Indeed, the different levels of LSs for the various segments (e.g. Fig. 7c) rise a question when the system is really clean and quiescent and also where the real photosphere of the light-dominating primary is. It is quite possible that the primary is significantly smaller than its lobe and that we see a surrounding structure of the circumstellar matter ("pseudophotosphere"). This may reconcile the two competing models for V Sge.

\subsection{Accreting white dwarf primary}

The characteristics of activity of $\mathrm{V}$ Sge in segments S3, S5, S7 (the semi-regular interchanging HSs and LSs, separated by rapid transitions) closely follow those observed in CVs of the VY Scl class. For example, clusters of LSs, sometimes replaced by extended intervals of almost stable brightness near the high state, commonly occur in V 442 Oph, S 193 (Garnavich \& Szkody 1988 and 1992) and KR Aur (Liller 1980). V Sge only differs in the level of the flat segments with respect to HSs in the active segments. HSs are slightly brighter than the adjacent flat segments in V Sge while brightness of HSs is equal to or lower than the level of seasons of the flat curve in S 193 and V 442 
Oph. Activity of the supersoft X-ray binary RX J0513.9 6951 (Alcock et al. 1996) is the most similar case to V Sge. The alternating HSs and LSs in RX J0513.9 quite resemble those in V Sge (segment S7), scaled 1.7 times down. Low states in the VY Scl-type CVs are caused by a temporary reduction of the mass transfer rate $\dot{m}$ (e.g. Shafter et al. 1985). The amount of matter outflowing from the loser strongly depends on the position of its photosphere with respect to the Roche limit and also on the current conditions near the $L_{1}$ point. It means that large changes of $\dot{m}$ can easily be obtained. Both the time scales and the course of the HS/LS behaviour in V Sge are consistent with the response of the mass accreting WD to variations of $\dot{m}$. This argument is strongly supported by the X-ray variations of V Sge during different optical states, reported by Greiner \& van Teeseling (1998).

The structure of HSs and LSs in V Sge can be resolved in some cases. The light curve, especially in S7, often displays a peak after recovery from LSs. This peak is followed by a decline of brightness through the episode of HS, which is completed by a rapid transition back into LS. On the other hand, a slow brightening through LS is seen in some cases. Livio \& Pringle (1994) and King \& Cannizzo (1998) offered an explanation for the occasional reduction of $\dot{m}$ in the VY Scl-type CVs by the magnetic field of a spot, appearing in the vicinity of the $L_{1}$ point of the late-type loser. It explains the episodic character of LSs but because it supposes solar-type spots it can work only for the cool stars with the convective outer layer (COL). However, in the case of V Sge it is very unlikely that the photosphere of its mass-losing secondary is cooler than about $7200 \mathrm{~K}$, when COL sets in. The mass ratio $q=3.8$ (HPSP) and the geometrical considerations (Paper I) imply its mass and radius typical for mid to late BV star. In addition, the course of HS/LS brings one even more conclusive evidence against the spot theory in V Sge. There is no reason that after disappearance of the spot the brightness should return to a higher level than before the episode of LS and give rise to the peak.

On the other hand, the course of HS/LS strongly resembles what would be expected in the case of instability when the system tries to find a steady state, but is forced to alternate between the high and low levels of brightness. High luminosity of V Sge even in LSs implies that $\dot{m}$ is still above the critical value, which does not allow the thermal instability to occur. It suggests that the disk remains in the hot state and allows to relate changes of the brightness and $\dot{m}$ directly (Smak 1989). The recent model for the irradiation-driven instability (hereafter IDI) of the outer layer of the loser (Wu et al. 1995) offers a promising mechanism. It makes use of the degree of filling of loser's lobe $x$, the mass transfer rate $y$, temperature of WD (accreting star) $\theta$ and indirectly also temperature $z$ of the loser's photosphere facing the accreting star (all parameters being normalized values). IDI allows to obtain double-valued $y$ for a distinct range of $x$ and $\theta$ (their Fig. 1b).
IDI solves the main observed characteristics of activity in V Sge. The two levels of HS and LS and the rapid transitions between them can be interpreted as a direct product of the two-level $\dot{m}$. Brightness of V Sge also often slowly declines through HS and increases again during LS, in accordance with the predicted course of the mass transfer rate in the IDI model. Moreover, this model allows $\dot{m}$ to vary even if the radius of the loser stays constant, because the growing dimensions of the disk (especially in the vertical direction) in epoch of the high $\dot{m}$ shield the loser from further irradiation. It makes the HS/LS transitions even more easy. Since the striking differences in the orbital modulation between HS and LS (HPSP, Patterson et al. 1998) imply large changes of the distribution of the circumstellar matter in $\mathrm{V}$ Sge, the variable degree of irradiation of the loser can be invoked. The promising mechanisms for the striking change of the orbital modulation are the modified disk with high rim (Meyer-Hofmeister et al. 1997; Schandl et al. 1997) and/or wind emanating from the luminous disk (Proga et al. 1998; Hachiya et al. 1998). They both can lead to a large increase of the dimension of the primary, when $\dot{m}$ enhances.

The facts, that the difference between the mean levels of the neighbouring active and flat segments is much smaller than the amplitude in the active segments (Fig. 5ab) and that the HSs are brighter than the levels of the adjoining flat segments (Fig. 7c) can be clarified, if the behaviour in the active segments is understood as a perturbation of relatively stable mass outflow from the loser, which occurs in the flat segments. The IDI model predicts that both double-valued $y$ and single-valued $y$ can co-exist and that the system can really alter between stable and unstable mass transfer, for example if $\theta$ varies.

Evolution of the character of the brightness variations from outbursts to HS/LS transitions is quantified by a gradual variation of skewness. It suggests that these seemingly different brightness variations in $V$ Sge are a product of a single mechanism and that the kind of activity just depends on the mean level of brightness in the given segment. We can offer an explanation for this evolution in the framework of IDI, if we include the long-term increase of $x$. The value of the mass outflow rate from the loser depends on its temperature $z$ (determined by $\theta$ ) and $x$. Only variations of $\theta$ suffice for modulation of $y$ inside a given segment. The value of $x$ then would determine the mean properties of activity inside the segment while a slow increase of $x$ from segment to segment may account for the evolution of activity.

The advantage of the model with the accreting WD and the irradiation- driven instability is that the activity and its evolution can be driven by just relatively small variations of the parameters of the mass-losing secondary, which are able to lead to large changes of $\dot{m}$. 


\section{Concluding remarks and implications}

We argue that the CV-like model, comprising the mass accreting WD primary, and variable mass transfer offer a viable explanation for the photometric activity of V Sge. Our analysis therefore brings an additional support to the model by Williams et al. (1986) and Patterson et al. (1998).

In this context, some previous findings should be briefly rediscussed. Absence of rotational disturbance during the primary eclipse in the spectra of Robertson et al. (1997) is not necessarily in contradiction with the model of the accreting WD primary. We can offer explanations in several ways: their data were obtained during HS (segment S7); the enhanced mass transfer in HS can lead to saturation of the amount of the circumstellar matter in the lobe of the primary, even with a possible formation of an extended envelope - the line profiles suggest a large amount of uneclipsed matter. Moreover, the emission structures in V Sge are highly variable, often persist just for several orbital cycles (Robertson et al. 1997; Patterson et al. 1998; Gies et al. 1998) and bear many links with the supersoft X-ray binary CAL 87 (Hutchings et al. 1998). The rotational disturbance is not visible even in some firmly established eclipsing CVs, lacking strong magnetic fields (e.g. Williams 1989; Warner 1995). Its absence therefore is not a conclusive argument against disk.

Also the orbital light curve of $\mathrm{V}$ Sge, mainly near LS, displays striking similarities to those of the eclipsing super-soft X-ray sources like CAL 87 and J0019.8 + 2156 . The modified disk around WD, expected for a high $\dot{m}$, is able to reproduce the main features (Meyer-Hofmeister et al. 1997; Schandl et al. 1997). It thus offers an alternative to the two-star model by Mader \& Shafter (1997).

We also may constitute a group of luminous CVlike systems with relatively long orbital periods $\left(P_{\text {orb }}>\right.$ 10 hours), which display large brightness variations on the time scale of days to weeks. It includes V Sge, RX J0513.9 - 6951 (Alcock et al. 1996) and RX J0019.9 + 2156 (Bartolini et al. 1996; Greiner \& Wenzel 1995). Since their changes often have a form of HSs and LSs, they are most likely invoked by variable $\dot{m}$. It suggests that there exists another region of the mass transfer instability, in addition to the range of $3<P_{\text {orb }}<4$ hours within which most VY Scl-type systems are observed (e.g. Warner 1995).

Acknowledgements. This research has made use of observations primarily from the AAVSO International database (Massachusetts, U.S.A), and also from the AFOEV database (operated at CDS, France). We thank the variable star observers worldwide whose decades of observations made this analysis possible. We thank Dr. R. Hudec for reading the manuscript and for valuable comments. V.Š. thanks Dr. S. Štefl for a discussion.

\section{References}

Alcock C., Allsman, R.A. Alves, et al., 1996, MNRAS 280, L49

Bartolini C., Guarnieri A., Piccioni A., Solmi L., 1996, 158th IAU Colloquium, Cataclysmic Variables and Related Objects, Evans A. and Wood J.H. (eds.). Kluwer Academic Publishers, p. 427

Eracleous M., Halpern J., Patterson J., 1991, ApJ 382, 290

Garnavich P.M., Szkody P., 1988, PASP 100, 1522

Garnavich P.M., Szkody P., 1992, JAAVSO 21, 81

Gies D.R., Shafter A.W., Wiggs M.S., 1998, AJ 115, 2566

Greiner J., Wenzel W., 1995, A\&A 294, L5

Greiner J., van Teeseling A., 1998, A\&A 339, L21

Hachiya M., Tajima Y., Fukue J., 1998, PASJ 50, 367

Harmanec P., 1994, in NATO ARW: The Impact of LongTerm Monitoring on Variable Star Research, Sterken C. and de Groot M. (eds.). Kluwer Academic Publishers, p. 55

Herbig G.H., Preston G.W., Smak J., Paczynski B., 1965, ApJ 141, 617 (HPSP)

Hjellming R.M., Hiltner W.A., 1963, ApJ 137, 1080

Hoard D.W., Wallerstein G., Willson L.A., 1996, PASP 108, 81

Hudec R., Meinunger L., 1977, Mitt. Veranderl. Sterne Sonneberg 7, 194

Hudec R., Wenzel W., 1986, A\&A 158, 396

Hutchings J.B., Crampton D., Cowley A.P., Schmidtke P.C., 1998, ApJ 502, 408

King A.R., Cannizzo J.K., 1998, ApJ 499, 348

Koch R.H., Corcoran M.F., Holenstein B.D., 1986, ApJ 306, 618

Liller M.H., 1980, AJ 85, 1092

Livio M., Pringle J.E., 1994, ApJ 427, 956

Lockley J.J., Eyres S.P.S., Wood J.H., 1997, MNRAS 287, L14

Mader J.A., Shafter A.W., 1997, PASP 109, 1351

Marsakova V.I., 1998, As. AP. Transact. 15, 143

Massey P., Lundstrom I., Stenholm B., 1984, PASP 96, 618

Mattei J., 1996, AAVSO International database (private communication)

Mennickent R.E., Vogt N., Sterken C., 1994, A\&AS 108, 237

Meyer-Hofmeister E., Schandl S., Meyer F., 1997, A\&A 321, 245

Patterson J., Kemp J., Shambrook A., et al., 1998, PASP 110, 380

Percy J.R., Jakate S.M., Matthews J.M., 1981, AJ 86, 53

Percy J.R., Fabro V.A., Keith D.W., 1985, J. AAVSO 14, 1

Proga D., Stone J.M., Drew J.E., 1998, MNRAS 295, 595

Richman H.R., Applegate J.H., Patterson J., 1994, PASP 106, 1075

Robertson J.W., Honeycutt R.K., Pier J.R., 1997, AJ 113, 787

Schandl S., Meyer-Hofmeister E., Meyer F., 1997, A\&A 318, 73

Schmutz W., 1991, IAU Symposium No. 143 Wolf-Rayet Stars and Interrelations with Other Massive Stars in Galaxies, van der Hucht K.A. and Hidayat B. (eds.), p. 39

Shafter A.W., Szkody P., Liebert J., Penning W.R., Bond H.E., Grauer A.D., 1985, ApJ 290, 707

Smak J., 1989, Acta Astron. 39, 317

Smak J., 1995, Acta Astron. 45, 361

Steiner J.E., Diaz M.P., 1998, PASP 110, 276

Stellingwerf R.F., 1978, ApJ 224, 953

Šimon V., 1996a, A\&A 309, 775 
Šimon V., 1996b, A\&AS 118, 421 (Paper I)

Šimon V., Mattei J.A., 1998, Proceedings of the 20th Stellar Conference of the Czech and Slovak Astronomical Institutes, Dušek J. and Zejda M. (eds.), Observatory and Planetarium Brno, p. 103

van den Heuvel E.P.J., Bhattacharya D., Nomoto K., Rappaport S.A., 1992, A\&A 262, 97

Warner B., 1995, Cataclysmic Variable Stars. Cambridge: Cambridge Univ. Press
Williams G.A., King A.R., Uomoto A.K., Hiltner W.A., 1986, MNRAS 219, 809

Williams R.E., 1989, AJ 97, 1752

Wood J.H., Lockley J.J., 1997, in IAU Colloq. 163, Accretion Phenomena and Related Outflows, Wickramasinghe T., Bicknell G.V. and Ferrario L. (eds.). ASP Conf. Ser. 121. San Francisco: ASP, p. 457

Wu K., Wickramasinghe D.T., Warner B., 1995, Publ. Astron. Soc. Aust. 12, 60 